\title{
Invariant regions and global existence of solutions for reaction-diffusion systems with a general full matrix
}

\section{Khaled Boukerrioua*}

\section{"Correspondence:}

khaledv2004@yahoo.fr

University of Guelma, Guelma, Algeria

\begin{abstract}
The aim of this study is to prove the global existence in time of solutions for reaction-diffusion systems. We make use of the appropriate techniques which are based on invariant regions and Lyapunov functional methods. We consider a full matrix of diffusion coefficients and we show the global existence of the solutions. MSC: $35 \mathrm{~K} 45 ; 35 \mathrm{~K} 57$
\end{abstract}

Keywords: global existence; reaction-diffusion systems; Lyapunov functional

\section{Introduction}

We are mainly interested in the global existence in time of solutions to a reaction-diffusion system of the form

$$
\begin{aligned}
& \left.\frac{\partial u}{\partial t}-a \Delta u-b \Delta v=\Pi-f(u, v)-\sigma u \text { in }\right] 0,+\infty[\times \Omega, \\
& \left.\frac{\partial v}{\partial t}-c \Delta u-d \Delta v=f(u, v)-\sigma v \text { in }\right] 0,+\infty[\times \Omega
\end{aligned}
$$

with the following boundary conditions:

$$
\left.\frac{\partial u}{\partial \eta}=\frac{\partial v}{\partial \eta}=0 \quad \text { in }\right] 0,+\infty[\times \partial \Omega
$$

and the initial data

$$
u(0, x)=u_{0}, \quad v(0, x)=v_{0} \quad \text { in } \Omega,
$$

where $\Omega$ is an open bounded domain in $\mathbb{R}^{n}$ with boundary $\partial \Omega$ of class $C^{1}, \frac{\partial}{\partial \eta}$ denotes the outward normal derivative on $\partial \Omega, \Delta$ denotes the Laplacian operator with respect to the $x$ variable, $a, b, c, d, \sigma$ are positive constants satisfying the condition $(b+c)^{2}<4 a d$, which reflects the parabolicity of the system and implies at the same time that the matrix of diffusion is positive definite, $\Pi \geq 0$. The eigenvalues $\lambda_{1}$ and $\lambda_{2}\left(\lambda_{1}<\lambda_{2}\right)$ of the matrix are positive. We assume that

$$
\lambda_{1}<a<d<\lambda_{2}<a+c,
$$

O2014 Boukerrioua; licensee Springer. This is an Open Access article distributed under the terms of the Creative Commons Attribution License (http://creativecommons.org/licenses/by/2.0), which permits unrestricted use, distribution, and reproduction in any medium, provided the original work is properly cited. 
and the initial data are assumed to be in the following region:

$$
\Sigma=\left\{\left(u_{0}, v_{0}\right) \in \mathbb{R}^{2} \text { such that } \frac{a-\lambda_{2}}{c} v_{0} \leq u_{0} \leq \frac{a-\lambda_{1}}{c} v_{0}\right\}
$$

For more details, one may consult [1].

The function $f$ is a nonnegative continuously differentiable function on $\Sigma$ such that

$$
f\left(\frac{a-\lambda_{2}}{c} \eta, \eta\right)=0 \text { and } f\left(\frac{a-\lambda_{1}}{c} \eta, \eta\right) \geq \frac{\Pi}{\left(1+\frac{a-\lambda_{1}}{c}\right)} \quad \text { for all } \eta \geq 0
$$

In addition we suppose that

$$
(\xi, \eta) \in \Sigma \quad \Longrightarrow \quad 0 \leq f(\xi, \eta) \leq \varphi(\xi)(1+\eta)^{\beta},
$$

where $\beta \geq 1$ and $\varphi$ is a nonnegative function of class $C(\mathbb{R})$ such that

$$
\lim _{\xi \rightarrow-\infty} \frac{\varphi(\xi)}{\xi}=0
$$

Melkemi et al. [2] established the existence of global solutions (eventually uniformly bounded in time) using a novel approach that involved the use of a Lyapunov function for system (1.1)-(1.4) when $c=b=0$. Along the same lines, Rebai [3] has proved the global existence of solutions for system (1.1)-(1.4), in the case $b=0, c>0$ (triangular matrix). The present investigation is a continuation of results obtained in [3]. Here, we follow the same reasoning as in [2], in the study of system (1.1)-(1.4), when $b>0, c>0$, that is, for a model that involves a general full matrix.

The components $u(t, x)$ and $v(t, x)$ represent either chemical concentrations or biological population densities and system (1.1)-(1.2) is a mathematical model describing various chemical and biological phenomena (see, e.g., Cussler [4]).

Remark 1 If $a<d$, then we have $\lambda_{1}<a<d<\lambda_{2}$. We note that the condition of parabolicity implies that $\operatorname{det}(A)=a d-b c>0$, where $A$ is the matrix of diffusion.

\section{Local existence and invariant regions}

Throughout the text we shall denote by \|\|$_{p}$ the norm in $L^{p}(\Omega)$, and by \|\|$_{\infty}$ the norm in $L^{\infty}(\Omega)$ or $C(\bar{\Omega})$.

For any initial data in $C(\bar{\Omega})$ or $\left.L^{p}(\Omega), p \in\right] 1,+\infty$ [, local existence and uniqueness of solutions to the initial value problem (1.1)-(1.4) follow from the basic existence theory for abstract semilinear differential equations (see Henry [5] and Pazy [6]). The solutions are classical on ]0; $T^{*}$ [, where $T^{*}$ denotes the eventual blowing-up time in $L^{\infty}(\Omega)$.

Furthermore, if $T^{*}<+\infty$, then

$$
\lim _{t \uparrow T^{*}}\left(\|u(t)\|_{\infty}+\|v(t)\|_{\infty}\right)=+\infty
$$

Therefore, if there exists a positive constant $C$ such that

$$
\left.\|u(t)\|_{\infty}+\|v(t)\|_{\infty} \leq C, \quad \forall t \in\right] 0, T^{*}[
$$

then $T^{*}=+\infty$. 
Multiplying (1.2) first through by $-\frac{a-\lambda_{2}}{c}$ and adding (1.1) and then by $\frac{a-\lambda_{1}}{c}$ and subtracting (1.1), we get

$$
\begin{aligned}
& \left.\frac{\partial w}{\partial t}-\lambda_{2} \Delta w=\Pi-\left(1+\frac{a-\lambda_{2}}{c}\right) F(w, z)-\sigma w \quad \text { in }\right] 0, T^{*}[\times \Omega, \\
& \left.\frac{\partial z}{\partial t}-\lambda_{1} \Delta z=-\Pi+\left(1+\frac{a-\lambda_{1}}{c}\right) F(w, z)-\sigma z \text { in }\right] 0, T^{*}[\times \Omega,
\end{aligned}
$$

with the boundary conditions

$$
\left.\frac{\partial w}{\partial \eta}=\frac{\partial z}{\partial \eta}=0 \text { in }\right] 0, T^{*}[\times \partial \Omega
$$

and the initial data

$$
w(0, x)=w_{0}(x), \quad z(0, x)=z_{0}(x) \quad \text { in } \Omega,
$$

where

$$
\begin{aligned}
& w(t, x)=u(t, x)-\frac{a-\lambda_{2}}{c} v(t, x), \\
& z(t, x)=-u(t, x)+\frac{a-\lambda_{1}}{c} v(t, x)
\end{aligned}
$$

for any $(t, x)$ in $] 0, T^{*}[\times \Omega$ and

$$
F(w, z)=f(u, v) \quad \text { for all }(u, v) \text { in } \Sigma .
$$

To prove that $\Sigma$ is an invariant region for system (1.1)-(1.4) it suffices to prove that the region

$$
\Sigma_{1}=\left\{\left(w_{0}, z_{0}\right) \in \mathbb{R}^{2} \text { such that } w_{0} \geq 0, z_{0} \geq 0\right\}
$$

is invariant for system (2.1)-(2.4).

Now, to prove that the region $\Sigma_{1}$ is invariant for system (2.1)-(2.4), it suffices to show that $\left(\Pi-\left(1+\frac{a-\lambda_{2}}{c}\right) F(0, z)\right) \geq 0$ for $z \geq 0$, and $\left(-\Pi+\left(1+\frac{a-\lambda_{1}}{c}\right) F(w, 0)\right) \geq 0$, for $w \geq 0$, see [7].

From (1.6), its clear that the region $\Sigma_{1}$ is invariant for system (2.1)-(2.4) and from (2.5) we have

$$
\begin{aligned}
& v(t, x)=\frac{c}{\lambda_{2}-\lambda_{1}}(w(t, x)+z(t, x)), \\
& u(t, x)=\frac{a-\lambda_{1}}{\lambda_{2}-\lambda_{1}} w(t, x)+\frac{a-\lambda_{2}}{\lambda_{2}-\lambda_{1}} z(t, x) .
\end{aligned}
$$

Remark 2 We note that if $(\xi, \eta) \in \Sigma$, then $\xi \in \mathbb{R}$ and $\eta \geq 0$.

\section{Existence of global solutions}

A simple application of the comparison theorem [7, Theorem 10.1] to system (2.1)-(2.4) implies that for any initial conditions $w_{0} \geq 0$ and $z_{0} \geq 0$, we have

$$
0 \leq w(t, x) \leq \max \left(\left\|w_{0}\right\|_{\infty}, \frac{\Pi}{\sigma}\right)=K
$$


To prove the global existence of the solutions of problem (1.1)-(1.4), one needs to prove it for problem (2.1)-(2.4). As regards this subject, it is well known that it suffices to derive a uniform estimate of $\left\|-\Pi+\left(1+\frac{a-\lambda_{1}}{c}\right) F(w, z)-\sigma z\right\|_{p}$ for some $p>\frac{n}{2}$, i.e.

$$
\left\|-\Pi+\left(1+\frac{a-\lambda_{1}}{c}\right) F(w, z)-\sigma z\right\|_{p} \leq C
$$

where $C$ is a nonnegative constant independent of $t$.

From the assumptions (1.7) and (1.8), we are led to establish the uniform boundedness of the $\|z\|_{p}$ on $] 0, T^{*}\left[\right.$ in order to get that of $\|z\|_{\infty}$ on $] 0, T^{*}[$.

For $p \geq 2$, we put

$$
\alpha=\frac{\left(\lambda_{2}-\lambda_{1}\right)^{2}}{4 \lambda_{1} \lambda_{2}}, \quad \alpha(p)=\frac{p \alpha+1}{p-1}, \quad M_{p}=K+\frac{\Pi}{\sigma \alpha(p)} .
$$

We firstly introduce the following lemmas, which are useful in our main results.

Lemma 1 Let $(w, z)$ be a solution of (2.1)-(2.4). Then

$$
\frac{d}{d t} \int_{\Omega} w d x+\left(1+\frac{a-\lambda_{2}}{c}\right) \int_{\Omega} F(w, z) d x+\sigma \int_{\Omega} w d x=\Pi|\Omega| .
$$

Proof We integrate both sides of (2.1), satisfied by $w$, which is positive and then we obtain

$$
\frac{d}{d t} \int_{\Omega} w d x=\Pi|\Omega|-\left(1+\frac{a-\lambda_{2}}{c}\right) \int_{\Omega} F(w, z) d x-\sigma \int_{\Omega} w d x
$$

Lemma 2 Assume that $p \geq 2$ and let

$$
G_{q}(t)=\int_{\Omega}\left[q w+\exp \left(-\frac{p-1}{p \alpha+1} \ln \left(\alpha(p)\left(M_{p}-w\right)\right)\right) z^{p}\right] d t
$$

where $(w, z)$ is the solution of (2.1)-(2.4) on $] 0, T^{*}[$. Then under the assumptions (1.7)-(1.8) there exist two positive constants $q>0$ and $s>0$ such that

$$
\frac{d}{d t} G_{q}(t) \leq-(p-1) \sigma G_{q}+s .
$$

Proof The proof is similar to that in Melkemi et al. [2].

Let

$$
h(w)=-\frac{p-1}{p \alpha+1} \ln \left(\alpha(p)\left(M_{p}-w\right)\right),
$$

then

$$
G_{q}(t)=q \int_{\Omega} w d x+N(t),
$$

where

$$
N(t)=\int_{\Omega} e^{h(w)} z^{p} d x .
$$


Differentiating $N(t)$ with respect to $t$ and using the Green formula one obtains

$$
\frac{d}{d t} N=H+S
$$

where

$$
\begin{aligned}
H= & -\lambda_{2} \int_{\Omega}\left(\left(h^{\prime}(w)\right)^{2}+h^{\prime \prime}(w)\right) e^{h(w)} z^{p}(\nabla w)^{2} d x \\
& -p\left(\lambda_{2}+\lambda_{1}\right) \int_{\Omega} h^{\prime}(w) e^{h(w)} z^{p-1} \nabla w \nabla z d x \\
& -\lambda_{1} \int_{\Omega} p(p-1) e^{h(w)} z^{p-2}(\nabla z)^{2} d x
\end{aligned}
$$

and

$$
\begin{aligned}
S= & \Pi \int_{\Omega} h^{\prime}(w) e^{h(w)} z^{p} d x \\
& +\int_{\Omega}\left[p z^{p-1}\left(1+\frac{a-\lambda_{1}}{c}\right) F(w, z)-\left(1+\frac{a-\lambda_{2}}{c}\right) h^{\prime}(w) z^{p} F(w, z)\right] e^{h(w)} d x \\
& -\sigma \int_{\Omega} h^{\prime}(w) w e^{h(w)} z^{p} d x-p \sigma \int_{\Omega} e^{h(w)} z^{p} d x-p \Pi \int_{\Omega} e^{h(w)} z^{p-1} d x .
\end{aligned}
$$

We observe that $H$ is given by

$$
H=-\int_{\Omega} Q e^{h(w)} d x
$$

where

$$
\begin{aligned}
Q= & \lambda_{2}\left(\left(h^{\prime}(w)\right)^{2}+h^{\prime \prime}(w)\right) z^{p}(\nabla w)^{2}+p\left(\lambda_{2}+\lambda_{1}\right) h^{\prime}(w) z^{p-1} \nabla w \nabla z \\
& +\lambda_{1} p(p-1) z^{p-2}(\nabla z)^{2}
\end{aligned}
$$

is a quadratic form with respect to $\nabla w$ and $\nabla z$, which is nonnegative if

$$
\left(p\left(\lambda_{2}+\lambda_{1}\right) h^{\prime}(w) z^{p-1}\right)^{2}-4 \lambda_{1} \lambda_{2} p(p-1)\left(\left(h^{\prime}(w)\right)^{2}+h^{\prime \prime}(w)\right) z^{2 p-2} \leq 0,
$$

and we have chosen $h(w)$ such that

$$
h^{\prime}(w)=\frac{1}{\alpha(p)\left(M_{p}-w\right)}, \quad h^{\prime \prime}(w)=\frac{\alpha(p)}{\left[\alpha(p)\left(M_{p}-w\right)\right]^{2}} .
$$

It is easy to see that the left-hand side of (3.13) can be written as

$$
\begin{aligned}
& 4 \lambda_{1} \lambda_{2} p z^{2 p-2}\left\{p\left[\alpha \frac{1}{\left(\alpha(p)\left(M_{p}-w\right)\right)^{2}}-\frac{\alpha(p)}{\left(\alpha(p)\left(M_{p}-w\right)\right)^{2}}\right]\right. \\
& \left.+\frac{1+\alpha(p)}{\left(\alpha(p)\left(M_{p}-w\right)\right)^{2}}\right\}=0,
\end{aligned}
$$


since

$$
p \alpha-p \alpha(p)+1+\alpha(p)=0
$$

the inequality (3.13) holds, $Q \geq 0$, and consequently

$$
H=-\int_{\Omega} Q e^{h(w)} d x \leq 0,
$$

and the second term $S$ can be estimated as

$$
\begin{aligned}
S \leq & \int_{\Omega}\left(\Pi h^{\prime}(w)-\sigma p\right) e^{h(w)} z^{p} d x \\
& +\int_{\Omega}\left[p z^{p-1}\left(1+\frac{a-\lambda_{1}}{c}\right) F(w, z)-h^{\prime}(w) z^{p}\left(1+\frac{a-\lambda_{2}}{c}\right) F(w, z)\right] e^{h(w)} d x \\
\leq & -(p-1) \sigma \int_{\Omega} e^{h(w)} z^{p} d x \\
& +\int_{\Omega}\left[\left(1+\frac{a-\lambda_{1}}{c}\right) p z^{p-1} F(w, z)-\left(1+\frac{a-\lambda_{2}}{c}\right) h^{\prime}(w) z^{p} F(w, z)\right] e^{h(w)} d x,
\end{aligned}
$$

since

$$
h^{\prime}(w)=\frac{1}{\alpha(p)\left(M_{p}-w\right)} \leq \frac{1}{\alpha(p)\left(M_{p}-K\right)}=\frac{\sigma}{\Pi} .
$$

On the other hand

$$
\begin{aligned}
& -h^{\prime}(w)=\frac{-1}{\alpha(p)\left(M_{p}-w\right)} \leq \frac{-1}{\alpha(p) M_{p}}, \\
& h(w) \leq \frac{-1}{\alpha(p)} \ln \frac{\Pi}{\sigma} .
\end{aligned}
$$

Taking into account the fact that $z \geq 0$, and using (3.19), we observe that

$$
\begin{aligned}
& p\left(1+\frac{a-\lambda_{1}}{c}\right) z^{p-1} F(w, z)-\left(1+\frac{a-\lambda_{2}}{c}\right) h^{\prime}(w) z^{p} F(w, z) \\
& \quad \leq\left(p\left(1+\frac{a-\lambda_{1}}{c}\right) z^{p-1}-\frac{1}{\alpha(p) M_{p}}\left(1+\frac{a-\lambda_{2}}{c}\right) z^{p}\right) F(w, z) .
\end{aligned}
$$

Then for $\eta_{0}=p\left(1+\frac{a-\lambda_{1}}{c}\right)\left(\frac{1}{\left(1+\frac{a-\lambda_{2}}{c}\right)}+1\right) \alpha(p) M_{p}>0$, and for $0 \leq \xi \leq K, \eta \geq \eta_{0}$, we have

$$
\begin{gathered}
\left(p\left(1+\frac{a-\lambda_{1}}{c}\right) \eta^{p-1}-\frac{1}{\alpha(p) M_{p}}\left(1+\frac{a-\lambda_{2}}{c}\right) \eta^{p}\right) F(\xi, \eta) \\
=\left[\frac{p\left(1+\frac{a-\lambda_{1}}{c}\right)}{\eta}-\frac{\left(1+\frac{a-\lambda_{2}}{c}\right)}{\alpha(p) M_{p}}\right] \eta^{p} F(\xi, \eta) \leq 0 .
\end{gathered}
$$

On the other hand, we deduce that the function

$$
(\xi, \eta) \rightarrow p\left(1+\frac{a-\lambda_{1}}{c}\right) \eta^{p-1}-\frac{1}{\alpha(p) M_{p}}\left(1+\frac{a-\lambda_{2}}{c}\right) \eta^{p}
$$


is bounded on the compact interval $\left[0, \eta_{0}\right]$; then there exists $c_{1}>0$ such that

$$
p\left(1+\frac{a-\lambda_{1}}{c}\right) z^{p-1} F(w, z)-\left(1+\frac{a-\lambda_{2}}{c}\right) h^{\prime}(w) z^{p} F(w, z) \leq c_{1} F(w, z) .
$$

From (3.17) and (3.20), we deduce immediately the following inequality:

$$
S \leq-(p-1) \sigma N+c_{1} \int_{\Omega} F(w, z) e^{h(w)} d x \leq-(p-1) \sigma N+c_{1} e^{\frac{-1}{\alpha(p)} \ln \frac{\Pi}{\sigma}} \int_{\Omega} F(w, z) d x,
$$

and putting

$$
q=\frac{c_{1}}{\left(1+\frac{a-\lambda_{2}}{c}\right)} e^{\frac{-1}{\alpha(p)} \ln \frac{\Pi}{\sigma}}
$$

by (3.3), we have

$$
S \leq-(p-1) \sigma N+q \Pi|\Omega|-q \frac{d}{d t} \int_{\Omega} w(t, x) d x
$$

and from (3.7), it follows that

$$
S \leq-(p-1) \sigma G_{q}+q((p-1) \sigma K+\Pi)|\Omega|-q \frac{d}{d t} \int_{\Omega} w(t, x) d x,
$$

and from (3.7) and (3.9), we conclude that

$$
\frac{d}{d t} G_{q} \leq-(p-1) \sigma G_{q}+s
$$

where

$$
s=q((p-1) \sigma K+\Pi)|\Omega| .
$$

Now we can establish the global existence and uniform boundedness of the solutions of (2.1)-(2.4).

Theorem 1 Under the assumptions (1.7) and (1.8), the solutions of (2.1)-(2.4) are global and uniformly bounded on $[0,+\infty[\times \Omega$.

Proof Multiplying the inequality (3.24) by $e^{(p-1) \sigma t}$ and then integrating, we deduce that there exists a positive constant $C>0$ independent of $t$, such that

$$
G_{q}(t) \leq C
$$

From (3.6), we observe that

$$
e^{h(w)} \geq e^{\frac{-1}{\alpha(p)} \ln \alpha(p) M_{p}},
$$


Boukerrioua Journal of Inequalities and Applications 2014, 2014:24

Page 8 of 10

http://www.journalofinequalitiesandapplications.com/content/2014/1/24

and it follows, for all $p \geq 2$, that

$$
\int_{\Omega} z^{p} d x \leq e^{\frac{1}{\alpha(p)} \ln \left(K \alpha(p)+\frac{\Pi}{\sigma}\right)} G_{q}(t) \leq C_{1}(p),
$$

where

$$
C_{1}(p)=C e^{\frac{1}{\alpha(p)} \ln \left(K \alpha(p)+\frac{\Pi}{\sigma}\right)}
$$

and as we select $p>\frac{n}{2}$ we can proceed to bound $\left\|-\Pi+\left(1+\frac{a-\lambda_{1}}{c}\right) F(w, z)-\sigma z\right\|_{p}$.

Let

$$
A=\max _{\xi_{0} \leq \xi \leq K_{1}} \varphi(\xi)
$$

where

$$
K_{1}=\frac{a-\lambda_{1}}{\lambda_{2}-\lambda_{1}} K
$$

and $\xi_{0}$ is such that

$$
\xi \leq \xi_{0} \quad \Longrightarrow \quad \varphi(\xi)<|\xi|,
$$

using (1.7), we deduce

$$
F(w, z)=f(u, v) \leq \varphi(u)(1+v)^{\beta},
$$

which implies

$$
\begin{aligned}
\int_{\Omega} F^{p}(w, z) d x & \leq \int_{\Omega}(\varphi(u))^{p}(1+v)^{\beta p} d x \\
& =\int_{u \leq \xi_{0}}(\varphi(u))^{p}(1+v)^{\beta p} d x+\int_{\xi_{0} \leq u}(\varphi(u))^{p}(1+v)^{\beta p} d x \\
& \leq \int_{u \leq \xi_{0}}|u|^{p}(1+v)^{\beta p} d x+A^{p} \int_{\xi_{0} \leq u}(1+v)^{\beta p} d x .
\end{aligned}
$$

From (2.7), we have

$$
\begin{aligned}
|u|^{p} & =\left|\frac{a-\lambda_{1}}{\lambda_{2}-\lambda_{1}} w(t, x)+\frac{a-\lambda_{2}}{\lambda_{2}-\lambda_{1}} z(t, x)\right|^{p} \leq\left(\frac{a-\lambda_{1}}{\lambda_{2}-\lambda_{1}} w(t, x)+\frac{\lambda_{2}-a}{\lambda_{2}-\lambda_{1}} z(t, x)\right)^{p} \\
& \leq\left(\frac{\lambda_{2}-a}{\lambda_{2}-\lambda_{1}}\right)^{p}(w(t, x)+z(t, x))^{p}
\end{aligned}
$$

then

$$
\begin{aligned}
\int_{\Omega} F^{p}(w, z) d x \leq & \int_{u \leq \xi_{0}}\left(\frac{\lambda_{2}-a}{\lambda_{2}-\lambda_{1}}\right)^{p}(w+z)^{p}\left(1+\frac{c}{\lambda_{2}-\lambda_{1}}(w+z)\right)^{\beta p} d x \\
& +A^{p} \int_{\xi_{0} \leq u}\left(1+\frac{c}{\lambda_{2}-\lambda_{1}}(w+z)\right)^{\beta p} d x
\end{aligned}
$$




$$
\begin{aligned}
& \leq \max \left(A^{p},\left(\frac{\lambda_{2}-a}{\lambda_{2}-\lambda_{1}}\right)^{p}\right)\left(\int_{u \leq \xi_{0}}(w+z)^{p}\left(1+\frac{c}{\lambda_{2}-\lambda_{1}}(w+z)\right)^{\beta p} d x\right. \\
& \left.\quad+\int_{\xi_{0} \leq u}\left(1+\frac{c}{\lambda_{2}-\lambda_{1}}(w+z)\right)^{\beta p} d x\right) \\
& \leq \max \left(A^{p},\left(\frac{\lambda_{2}-a}{\lambda_{2}-\lambda_{1}}\right)^{p}\right)\left(\int_{\Omega}(w+z)^{p}\left(1+\frac{c}{\lambda_{2}-\lambda_{1}}(w+z)\right)^{\beta p} d x\right. \\
& \left.\quad+\int_{\Omega}\left(1+\frac{c}{\lambda_{2}-\lambda_{1}}(w+z)\right)^{\beta p} d x\right), \\
& \int_{\Omega}(w+z)^{p}\left(1+\frac{c}{\lambda_{2}-\lambda_{1}}(w+z)\right)^{\beta p} d x \\
& \leq 2^{\beta p-1}\left(\int_{\Omega}(w+z)^{p}+\left(\frac{c}{\lambda_{2}-\lambda_{1}}\right)^{\beta p}(w+z)^{(\beta+1) p} d x\right) \\
& \leq 2^{(\beta+1) p-2}\left(K^{P}|\Omega|+C_{1}(p)\right)+2^{(2 \beta+1) p-2}\left(\frac{c}{\lambda_{2}-\lambda_{1}}\right)^{\beta p}\left(K^{(\beta+1) p}|\Omega|+C_{1}((\beta+1) p)\right) \\
& =C_{2}(\beta, p, K, \Omega), \\
& \int_{\Omega}\left(1+\frac{c}{\lambda_{2}-\lambda_{1}}(w+z)\right)^{\beta p} d x \\
& \leq 2^{\beta p-1}\left(|\Omega|+\left(\frac{c}{\lambda_{2}-\lambda_{1}}\right)^{\beta p} \times 2^{\beta p-1}\left(K^{\beta p}|\Omega|+C_{1}(\beta p)\right)\right) \\
& =C_{3}(\beta, p, K, \Omega) .
\end{aligned}
$$

\section{Consequently}

$$
\int_{\Omega} F^{p}(w, z) d x \leq C_{4}(A, \beta, p, K, \Omega) .
$$

Finally

$$
\begin{aligned}
\left\|-\Pi+\frac{a-\lambda_{1}}{c} F(w, z)-\sigma z\right\|_{p} & =\left\|\frac{a-\lambda_{1}}{c} F(w, z)-(\sigma z+\Pi)\right\|_{p} \\
& \leq \frac{a-\lambda_{1}}{c}\|F(w, z)\|_{p}+\sigma\|z\|_{p}+\Pi|\Omega| \\
& \leq \frac{a-\lambda_{1}}{c} \sqrt[p]{C_{4}(A, \beta, p, K)}+\sigma \sqrt[p]{C_{1}(p)}+\Pi|\Omega| \\
& =C_{5}(A, \beta, p, K, \Omega, \sigma) .
\end{aligned}
$$

Using the regularity results for the solutions of the parabolic equations in [5], we conclude that the solutions of problem (2.1)-(2.4) are uniformly bounded on $[0,+\infty[\times \Omega$.

By (2.7), it is easy to see that the solutions of problem (1.1)-(1.4) are also uniformly bounded on $[0,+\infty[\times \Omega$.

Remark 3 Because $0 \leq w(t, x) \leq K$ and $z(t, x) \geq 0$, we deduce that

$$
-\infty \leq u(t, x) \leq \frac{a-\lambda_{1}}{\lambda_{2}-\lambda_{1}} K=K_{1}
$$


Remark 4 We note that $\frac{a-\lambda_{2}}{\lambda_{2}-\lambda_{1}}<0$ and $\frac{\lambda_{2}-a}{\lambda_{2}-\lambda_{1}} \geq \frac{a-\lambda_{1}}{\lambda_{2}-\lambda_{1}}$, because $\lambda_{2}+\lambda_{1}=a+d$ and $d>a$.

We conclude by noting that the study of the global existence of strongly coupled systems has been a major development, and several articles are devoted to this subject. In our opinion, many other systems with non-constant diffusion matrix which are in the actual scope of the results previously given, should be taken in consideration and studied with more interest.

\section{Competing interests}

The author declares that they have no competing interests.

\section{Acknowledgements}

The author would like to thank the anonymous referee for his/her valuable suggestions.

Received: 30 July 2013 Accepted: 26 December 2013 Published: 23 Jan 2014

\section{References}

1. Kouachi, S: Invariant regions and global existence of solutions for reaction-diffusion systems with a full matrix of diffusion coefficients and nonhomogeneous boundary conditions. Georgian Math. J. 11(2), 349-359 (2004)

2. Melkemi, L, Mokrane, AZ, Youkana, A: On the uniform boundedness of the solutions of systems of reaction-diffusion equations. Electron. J. Qual. Theory Differ. Equ. 2005, 24 (2005)

3. Rebai, B: Global classical solutions for reaction-diffusion systems with a triangular matrix of diffusion coefficients. Electron. J. Differ. Equ. 2011, 99 (2011)

4. Cussler, EL: Multicomponent Diffusion. Chemical Engineering Monographs, vol. 3. Elsevier, Amsterdam (1976)

5. Henry, D: Geometric Theory of Semi-Linear Parabolic Equations. Lecture Notes in Mathematics, vol. 840. Springer, New York (1984)

6. Pazy, A: Semigroups of Linear Operators and Applications to Partial Differential Equations. Applied Mathematical Sciences, vol. 44. Springer, New York (1983)

7. Smoller, J: Shock Waves and Reaction-Diffusion Equations. Springer, New York (1983)

10.1186/1029-242X-2014-24

Cite this article as: Boukerrioua: Invariant regions and global existence of solutions for reaction-diffusion systems

with a general full matrix. Journal of Inequalities and Applications 2014, 2014:24

\section{Submit your manuscript to a SpringerOpen ${ }^{\circ}$ journal and benefit from:}

- Convenient online submission

- Rigorous peer review

- Immediate publication on acceptance

- Open access: articles freely available online

- High visibility within the field

- Retaining the copyright to your article 